pregnancies (including the work of Mayer, Glenister, Kirby, and others) but nothing on placental structure and function, foetal development, and the like. The bibliography contains useful references on gestation, but barely half a dozen text and list references correspond.

Finally, there is the contribution on "Chromosomes and Chromosomal Evolution in Mammals", by Robert Matthey. It is a revised version of an account that appeared in volume twelve (1954) and deals expertly with sex chromosomes, the sex ehromatin and the Lyon hypothesis (half a page), chromosomal evolution (with lists of diploid numbers) and chromosomal polymorphism. For sources of data before 1958, the reader is offered six key references, but none for the numerous more recent text citations.

In spite of its shortcomings, this is an impressive publication and cannot fail to be accepted as the standard reference in most of the fields that it covers.

\section{R. Austin}

amount of overlap in each. Important topics such as controlled burning and grazing are considered in a couple of pages each.

In spite of these criticisms, however, I found some comprehensive sections on aerial census techniques, in which systematic and random methods are compared; quantity and quality of diet; reproduction, in which a population of sheep is taken as an example; anatomical and micro. biological studies of digestive tracts; immobilization, which is covered by two papers, and epizootiology.

At the end, Petrides provides some helpful conversion factors for productivity measurements.

In conclusion, this is a useful book but of uneven quality. It gives the impression that it has suffered from unequal effort spent on the various sections and from the change in editorship during its gestation.

Christopher R. Field

\section{FROM GRASS TO MEAT}

\section{A Practical Guide to the Study of the Productivity of Large Herbivores}

(International Biological Programme Handbook No. 7.) Edited by Frank B. Golley and Helmut K. Buechner. Pp. xii +308 . (Blackwell (Scientific): Oxford and Edinburgh, 1968.) $40 s$.

THIs book should be read by scientists before embarking on studies of the productivity of large herbivores. It is of most value in giving direction to the work of research teams and is less suitable for use in the field.

A wide range of interests are covered which are roughly divided into energy flow and its components, and management for secondary production. The latter deals with animal husbandry and handling, physiology, reproduction and nutrition. There is also a paper on epizootiology and a section on habitat manipulation involving a number of papers.

Throughout the book there is a wealth of information and advice on techniques which should help reduce unnecessary and wasteful effort. A great deal of common sense is written, but the quality of the papers is varied so that some appear directed at the student level while others are fairly technical.

This handbook on methodology was conceived at an IBP symposium in Aberdeen and Cambridge in 1965, but was not published until almost three years later. As a result it suffers from not keeping abreast with more recent work. Nevertheless, there is little excuse for the absence of reference to some important contributions, such as the work of D. R. M. Stewart on the diet of ungulates by faecal analyses or the brief reference to the work of R. M. Laws on population dynamics of large herbivores. Both of these workers attended the original meeting.

Some of the papers are presented without fully appreciating that they are not necessarily vectors for the description of original work, but are intended primarily to advise on the best methods to use. 'Thus, a brief study of an elephant population in Uganda is used to illustrate secondary productivity and energy relationships when more thorough studies have been made on other populations.

In other cases an author has written on a subject in which he has secondary interests. Harthoorn gives a comprehensive paper on the chemical restraint of herbivores, in which he makes the use of drugs sound delightfully easy, but his paper on animal husbandry is vague and disappointing in comparison.

In contrast to this situation, the section on habitat manipulation requires consolidation. There are eleven papers averaging four to five pages in length with a certain

\section{WHITE ANT BIOLOGY \\ Biology of Termites}

Vol. 1. Edited by Kumar Krishna and Francis M. Weesner. Pp. xiii + 598. (Academic Press: New York and London, April, 1969.) 298s.

THE price and weight of this book at once imply multiple authorship. There are indeed fifteen contributors, each an active investigator in the field, who contribute among them seventeen chapters each with forty to a hundred or more references. This first volume contains general or comparative accounts of external and internal anatomy, behaviour, food exchanges within the colony, water relations, culture techniques, biochemistry, the spread of termite species by man, and the relations between termites and fungi and between termites and termite guests. Volume two is to deal with "the taxonomy and general biology of the termites of the different zoogeographical regions". It seems a pity that no space was found in the first volume for a general account of the importance of termites as primary consumers, for there is surely much to be said here that applies to all regions. Unfortunately, the introductory chapter (by Krishna) does not deal with these generalities about termites but, after outlining a classification of Isoptera which is not used by several of the authors, it passes into a history of termite research. Some other chapters have the same failing. While a history of termite research may sometimes be illuminating, this is not always so, and the subject of the book is biology, not history.

The individual chapters are all good, but as in most books of this form there are gaps and overlaps between them. The earlier chapters on anatomy mostly treat function as well as structure, though the reader who wonders how soldier termites use their strangely contorted mandibles (chapter two) has to wait till chapter ten (on polymorphism) for an answer. Perhaps the biggest overlap is in the realm of behaviour. Stuart and Nutting both deal with flight and pairing but from rather different viewpoints, and with a stimulating degree of disagreement over the stimuli maintaining tandem behaviour. Pheromones and responses to them appear in Moore's chapter on biochemistry, and again the overlap with Stuart's treatment of them is beneficial.

The complex matter of caste determination is covered in two chapters. The first, by Miller, deals very clearly with the situation in lower termites, leaning heavily on Kalotermes. Noirot's chapter follows caste formation in Termitidae in which a stable worker caste occurs. The following five chapters-on culture methods (Becker), feeding relationships (McMahan), biochemical studies (Moore), water relations (Collins) and species introduced by man (Gay) - go well outside a narrow interpretation of their topic. The last two chapters may, however, be the most interesting to the non-specialist. Sands's review of 